\title{
The strong solutions for a class of fluid-particle interaction non-Newtonian models
}

\author{
Yukun Song ${ }^{1 *}$, Heyuan Wang ${ }^{1}$, Yang Chen ${ }^{1}$ and Yunliang Zhang ${ }^{2}$
}

\section{"Correspondence:}

songyukun8@163.com

'College of Science, Liaoning

University of Technology, Jinzhou,

Liaoning 121001, P.R. China

Full list of author information is

available at the end of the article

\begin{abstract}
The aim of this paper is to discuss the existence and uniqueness for a class of fluid-particle interaction non-Newtonian models which describe the evolution of particles dispersed in a viscous compressible non-Newtonian fluid. The strong nonlinearity of the system and the singularity of the viscosity term bring about difficulties. Also, we admit an initial vacuum.
\end{abstract}

Keywords: strong solution; fluid-particle interaction model; non-Newtonian fluid; vacuum

\section{Introduction}

In this paper, we consider the following non-Newtonian fluids system:

$$
\left\{\begin{array}{l}
\rho_{t}+(\rho u)_{x}=0 \\
(\rho u)_{t}+\left(\rho u^{2}\right)_{x}-\lambda\left(\left|u_{x}\right|^{p-2} u_{x}\right)_{x}+(P+\eta)_{x}=-(\eta+\beta \rho) \Phi_{x}, \quad(x, t) \in \Omega_{T} \\
\eta_{t}+\left(\eta\left(u-\Phi_{x}\right)\right)_{x}=\eta_{x x}
\end{array}\right.
$$

with the initial conditions

$$
\left.(\rho, u, \eta)\right|_{t=0}=\left(\rho_{0}, u_{0}, \eta_{0}\right), \quad x \in \Omega
$$

together with the no-slip boundary conditions for the velocity and the no-flux condition for the density of particles,

$$
\left.u\right|_{\partial \Omega}=\left.\left(\eta_{x}+\eta \Phi_{x}\right)\right|_{\partial \Omega}=0, \quad t \in[0, T]
$$

where $\rho, u, \eta, P(\rho)=a \rho^{\gamma}, \Phi(x)$ denote the fluid density, velocity, the density of particles in the mixture, the pressure, and the external potential, respectively, $a>0, \gamma>1, \frac{4}{3}<p<2$. $\lambda>0$ is the viscosity coefficient and $\beta \neq 0$ is a constant, $\Omega$ is a one-dimensional bounded interval, and for simplicity we only consider $\Omega=(0,1), \Omega_{T}=\Omega \times[0, T]$.

Fluid-particle interaction models arise in a lot of industrial procedures such as the analysis of the sedimentation phenomenon. These procedures find their applications in

(c) 2016 Song et al. This article is distributed under the terms of the Creative Commons Attribution 4.0 International License (http://creativecommons.org/licenses/by/4.0/), which permits unrestricted use, distribution, and reproduction in any medium, provided you give appropriate credit to the original author(s) and the source, provide a link to the Creative Commons license, and indicate if changes were made. 
biotechnology, medicine, mineral processing, and chemical engineering [1-4]. Such interaction systems are also used in combustion theory, when modeling Diesel engines or rocket propulsors $[5,6]$.

The coupled microscopic/macroscopic models describe the evolution of particles dispersed in a fluid. The system consists in a Vlasov-Fokker-Planck equation to describe the microscopic motion of the particles coupled to the equations for the fluid. Generally speaking, at the microscopic scale, the cloud of particles is described by its distribution function $f(x, \xi, t)$, a solution to a Vlasov-Fokker-Planck equation. The fluid, on the other hand, is modeled by macroscopic quantities, namely its density $\rho(x, t) \geq 0$ and its velocity field $u(x, t)$. If the fluid is compressible and isentropic, then $(\rho, u)$ solves the compressible Euler (inviscid case) or Navier-Stokes system (viscous case) of equations. With the dynamic viscosity terms taken into consideration, Carrillo et al. [7] discussed the following system:

$$
\left\{\begin{array}{l}
\rho_{t}+\operatorname{div}(\rho u)=0 \\
(\rho u)_{t}+\operatorname{div}(\rho u \otimes u)+\nabla(P(\rho)+\eta)-\mu \Delta u-\lambda \nabla \operatorname{div} u=-(\eta+\beta \rho) \nabla \Phi \\
\eta_{t}+\operatorname{div}(\eta(u-\nabla \Phi))-\Delta \eta=0
\end{array}\right.
$$

They obtained the global existence and asymptotic behavior of the weak solutions to (1.4) following the framework of Lions [8] and Feireisl et al. [9, 10]. In addition, Mellet and Vasseur [11] proved the global existence of weak solutions of equations by using the entropy method on the asymptotic regime corresponding to a strong drag force and strong Brownian motion.

In recent years, there has been an increasing recognition of the importance of nonNewtonian flow characteristics displayed by most materials encountered in everyday life, both in nature (gums, proteins, biological fluids such as blood, synovial fluid, etc.) and in technology (polymers and plastics, emulsions, slurries, etc.) (see [12]). Since there has been much research in the field of non-Newtonian flows, both theoretically and experimentally, let us briefly recall the related results in the literature. Bellout et al. [13] studied the non-Newtonian fluids for space periodic problems and showed the Young measurevalued solutions. In [14], Guo and Zhu investigated the partial regularity of the generalized solutions to the modified Navier-Stokes equations which describes the dynamics of the incompressible monopolar non-Newtonian fluids. Zhao et al. [15] constructed the trajectory attractor and global attractor for an autonomous two-dimensional non-Newtonian fluid. Yuan and $\mathrm{Xu}$ [16] obtained the existence and uniqueness of solutions for a class of nonNewtonian fluids with singularity and vacuum. For other results we may refer to [17-23].

It is worth mentioning that most recently, Fang et al. [24] got the global existence of classical solutions of (1.4) in dimension one, namely $p=2$ in (1.1). Compared with the work of [24], the strong nonlinearity of (1.1) brings us new difficulties in getting the upper bound of $\rho$, which plays an important role throughout their proof. The second equation of (1.1) is always with singularity and brings us another difficulty. Motivated by Cho et al.'s $[25,26]$ work on the Navier-Stokes equations, we establish local existence and uniqueness of strong solutions of (1.1).

Throughout the paper we assume that $a=\lambda=1$ for simplicity. In the following sections, we will use simplified notations for standard Sobolev spaces and Bochner spaces, such as $L^{p}=L^{p}(\Omega), H_{0}^{1}=H_{0}^{1}(\Omega), C\left([0, T] ; H^{1}\right)=C\left([0, T] ; H^{1}(\Omega)\right)$. 


\subsection{Main results}

Theorem 1.1 Let $\Phi \in C^{2}(\Omega)$ and assume that the initial data $\left(\rho_{0}, u_{0}, \eta_{0}\right)$ satisfy the following conditions:

$$
0 \leq \rho_{0} \in H^{1}(\Omega), \quad u_{0} \in H_{0}^{1}(\Omega) \cap H^{2}(\Omega), \quad \eta_{0} \in H^{2}(\Omega),
$$

and the compatibility condition

$$
\left(\left|u_{0 x}\right|^{p-2} u_{0 x}\right)_{x}-\left(P\left(\rho_{0}\right)+\eta_{0}\right)_{x}-\eta_{0} \Phi_{x}=\rho_{0}\left(g+\beta \Phi_{x}\right),
$$

for some $g \in L^{2}(\Omega)$. Then there exist a $T_{*} \in(0,+\infty)$ and a unique strong solution $(\rho, u, \eta)$ to (1.1)-(1.3) such that

$$
\begin{aligned}
& \rho \in L^{\infty}\left(0, T_{*} ; H^{1}(\Omega)\right), \quad \rho_{t} \in L^{\infty}\left(0, T_{*} ; L^{2}(\Omega)\right), \\
& u \in L^{\infty}\left(0, T_{*} ; W_{0}^{1, p}(\Omega) \cap H^{2}(\Omega)\right), \quad u_{t} \in L^{2}\left(0, T_{*} ; H_{0}^{1}(\Omega)\right), \\
& \eta \in L^{\infty}\left(0, T_{*} ; H^{2}(\Omega)\right), \quad \eta_{t} \in L^{\infty}\left(0, T_{*} ; H^{1}(\Omega)\right), \\
& \sqrt{\rho} u_{t} \in L^{\infty}\left(0, T_{*} ; L^{2}(\Omega)\right), \quad\left(\left|u_{*}\right|^{p-2} u_{*}\right)_{x} \in L^{2}\left(0, T_{*} ; L^{2}(\Omega)\right) .
\end{aligned}
$$

\section{A priori estimates for smooth solutions}

In this section, we will prove the local existence of strong solutions. Because equation $(1.1)_{2}$ always possesses a singularity, we overcome this difficulty by a regularized process, then taking the limiting process back to the original problem. First of all, we consider the following system:

$$
\begin{aligned}
& \rho_{t}+(\rho u)_{x}=0 \\
& (\rho u)_{t}+\left(\rho u^{2}\right)_{x}-\left[\left(\frac{\varepsilon u_{x}^{2}+1}{u_{x}^{2}+\varepsilon}\right)^{\frac{2-p}{2}} u_{x}\right]_{x}+(P+\eta)_{x}=-(\eta+\beta \rho) \Phi_{x} \\
& \eta_{t}+\left(\eta\left(u-\Phi_{x}\right)\right)_{x}=\eta_{x x}
\end{aligned}
$$

with the initial and boundary conditions,

$$
\begin{array}{ll}
\left.(\rho, u, \eta)\right|_{t=0}=\left(\rho_{0}, u_{0}, \eta_{0}\right), & x \in \Omega, \\
\left.u\right|_{\partial \Omega}=\left.\left(\eta_{x}+\eta \Phi_{x}\right)\right|_{\partial \Omega}=0, & t \in[0, T],
\end{array}
$$

and $u_{0} \in H_{0}^{1}(\Omega) \cap H^{2}(\Omega)$ is the smooth solution of the boundary value problem

$$
\left\{\begin{array}{l}
{\left[\left(\frac{\varepsilon u_{0 x}^{2}+1}{u_{0 x}^{2}+\varepsilon}\right)^{\frac{2-p}{2}} u_{0 x}\right]_{x}-\left(P\left(\rho_{0}\right)+\eta_{0}\right)_{x}-\eta_{0} \Phi_{x}=\rho_{0}\left(g+\beta \Phi_{x}\right),} \\
u_{0}(0)=u_{0}(1)=0
\end{array}\right.
$$

By using the iterative method step by step, the nonlinear coupled system admits a smooth solution (see Section 3). Provided that $(\rho, u, \eta)$ is a smooth solution of (2.1)-(2.5) and $\rho_{0} \geq$ $\delta$, where $0<\delta \ll 1$ is a positive number. We denote $M_{0}=1+\mu_{0}+\mu_{0}^{-1}+\left|\rho_{0}\right|_{H^{1}}+|g|_{L^{2}}$.

First we obtain the estimate of $\left|u_{0 x x}\right|_{L^{2}}$. From (2.6), we have

$$
u_{0 x x}=\left(\frac{\varepsilon u_{0 x}^{2}+1}{u_{0 x}^{2}+\varepsilon}\right)^{\frac{p}{2}} \frac{\left(u_{0 x}^{2}+\varepsilon\right)^{2}\left[\left(P\left(\rho_{0}\right)+\eta_{0}\right)_{x}+\eta_{0} \Phi_{x}+\rho_{0}\left(g+\beta \Phi_{x}\right)\right]}{\left(\varepsilon u_{0 x}^{2}+1\right)\left(u_{0 x}^{2}+\varepsilon\right)-(2-p)\left(1-\varepsilon^{2}\right) u_{0 x}^{2}} .
$$


Then

$$
\begin{aligned}
\left|u_{0 x x}\right|_{L^{2}} \leq & \frac{1}{p-1}\left|\left(\frac{u_{0 x}^{2}+\varepsilon}{\varepsilon u_{0 x}^{2}+1}\right)^{1-\frac{p}{2}}\right|_{L^{\infty}}\left|\left(P\left(\rho_{0}\right)+\eta_{0}\right)_{x}+\eta_{0} \Phi_{x}+\rho_{0}\left(g+\beta \Phi_{x}\right)\right|_{L^{2}} \\
\leq & \frac{1}{p-1}\left(\left|u_{0 x}\right|_{L^{\infty}}^{2}+1\right)^{1-\frac{p}{2}}\left(\left|\left(P\left(\rho_{0}\right)+\eta_{0}\right)_{x}+\eta_{0} \Phi_{x}+\rho_{0}\left(g+\beta \Phi_{x}\right)\right|_{L^{2}}\right) \\
\leq & \frac{1}{p-1}\left(\left|u_{0 x x}\right|_{L^{2}}^{2}+1\right)^{1-\frac{p}{2}}\left(a \gamma\left|\rho_{0}\right|_{L^{\infty}}^{\gamma-1}\left|\rho_{0 x}\right|_{L^{2}}+\left|\eta_{0 x}\right|_{L^{2}}+\left|\eta_{0}\right|_{L^{\infty}}\left|\Phi_{x}\right|_{L^{2}}\right. \\
& \left.+\left|\rho_{0}\right|_{L^{\infty}}|g|_{L^{2}}+\beta\left|\rho_{0}\right|_{L^{\infty}}\left|\Phi_{x}\right|_{L^{2}}\right) .
\end{aligned}
$$

Using Young's inequality, we have

$$
\left|u_{0 x x}\right|_{L^{2}} \leq C
$$

where $C$ is a positive constant, depending only on $M_{0}$.

Next, we introduce an auxiliary function,

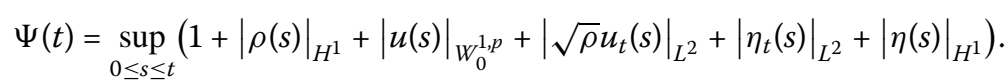

Then we estimate each term of $\Psi(t)$ in terms of some integrals of $\Psi(t)$, apply arguments of Gronwall-type, and thus prove that $\Psi(t)$ is locally bounded.

\subsection{Estimate for $|\rho|_{H^{1}}$}

First we need the following estimates for $u$ and $\eta$. By virtue of (2.2)

$$
\left[\left(\frac{\varepsilon u_{x}^{2}+1}{u_{x}^{2}+\varepsilon}\right)^{\frac{2-p}{2}} u_{x}\right]_{x}=\rho u_{t}+\rho u u_{x}+(P+\eta)_{x}+(\eta+\beta \rho) \Phi_{x} .
$$

Then, we have

$$
\left|u_{x x}\right| \leq \frac{1}{p-1}\left(u_{x}^{2}+\varepsilon\right)^{1-\frac{p}{2}}\left|\rho u_{t}+\rho u u_{x}+(P+\eta)_{x}+(\eta+\beta \rho) \Phi_{x}\right| .
$$

Taking the $L^{2}$ norm and using Young's inequality, we get

$$
\begin{aligned}
\left|u_{x x}\right|_{L^{2}}^{p-1} \leq & C\left(1+\left|\rho u_{t}\right|_{L^{2}}+\left|\rho u u_{x}\right|_{L^{2}}+\left|(P+\eta)_{x}\right|_{L^{2}}+\left|(\eta+\beta \rho) \Phi_{x}\right|_{L^{2}}\right) \\
\leq & C\left(1+|\rho|_{L^{\infty}}^{\frac{1}{2}}\left|\sqrt{\rho} u_{t}\right|_{L^{2}}+|\rho|_{L^{\infty}}|u|_{L^{\infty}}\left|u_{x}\right|_{L^{p}}^{\frac{p}{2}}\left|u_{x}\right|_{L^{\infty}}^{1-\frac{p}{2}}\right. \\
& \left.+|\rho|_{L^{\infty}}^{\gamma-1}\left|\rho_{x}\right|_{L^{2}}+\left|\eta_{x}\right|_{L^{2}}+|\eta|_{L^{\infty}}\left|\Phi_{x}\right|_{L^{2}}+|\rho|_{L^{\infty}}\left|\Phi_{x}\right|_{L^{2}}\right) \\
\leq & C\left[1+|\rho|_{L^{\infty}}^{\frac{1}{2}}\left|\sqrt{\rho} u_{t}\right|_{L^{2}}+\left(|\rho|_{L^{\infty}}|u|_{L^{\infty}}\left|u_{x}\right|_{L^{p}}^{\frac{p}{2}}\right)^{\frac{2(p-1)}{3 p-4}}+|\rho|_{L^{\infty}}^{\gamma-1}\left|\rho_{x}\right|_{L^{2}}\right. \\
& \left.+\left|\eta_{x}\right|_{L^{2}}+|\eta|_{L^{\infty}}\left|\Phi_{x}\right|_{L^{2}}+|\rho|_{L^{\infty}}\left|\Phi_{x}\right|_{L^{2}}\right]+\frac{1}{2}\left|u_{x x}\right|_{L^{2}}^{p-1} \\
\leq & C \Psi^{\frac{(4+p)(p-1)}{3 p-4} \gamma}(t)+\frac{1}{2}\left|u_{x x}\right|_{L^{2}}^{p-1} .
\end{aligned}
$$

Hence, we deduce that

$$
\left|u_{x x}\right|_{L^{2}} \leq C \Psi^{\frac{6 \gamma}{3 p-4}}(t)
$$


From (2.3), taking the $L^{2}$ norm, we get

$$
\begin{aligned}
\left|\eta_{x x}\right|_{L^{2}} & \leq\left|\eta_{t}+\left(\eta\left(u-\Phi_{x}\right)\right)_{x}\right|_{L^{2}} \\
& \leq\left|\eta_{t}\right|_{L^{2}}+\left|\eta_{x}\right|_{L^{2}}|u|_{L^{\infty}}+\left|\eta_{x}\right|_{L^{2}}\left|\Phi_{x}\right|_{L^{\infty}}+|\eta|_{L^{2}}\left|u_{x x}\right|_{L^{2}}+|\eta|_{L^{\infty}}\left|\Phi_{x x}\right|_{L^{2}} \\
& \leq C \Psi^{\frac{8 \gamma}{3 p-4}}(t) .
\end{aligned}
$$

Multiplying (2.1) by $\rho$, integrating over $\Omega$, we have

$$
\frac{1}{2} \frac{\mathrm{d}}{\mathrm{d} t} \int_{\Omega}|\rho|^{2} \mathrm{~d} s+\int_{\Omega}(\rho u)_{x} \rho \mathrm{d} x=0
$$

Integrating by parts, using the Sobolev inequality, we deduce that

$$
\frac{\mathrm{d}}{\mathrm{d} t}|\rho(t)|_{L^{2}}^{2} \leq \int_{\Omega}\left|u_{x}\right||\rho|^{2} \mathrm{~d} x \leq\left|u_{x x}\right|_{L^{2}}|\rho|_{L^{2}}^{2}
$$

Differentiating $(1.1)_{1}$ with respect to $x$, and multiplying it by $\rho_{x}$, integrating over $\Omega$, and using the Sobolev inequality, we have

$$
\begin{aligned}
\frac{\mathrm{d}}{\mathrm{d} t} \int_{\Omega}\left|\rho_{x}\right|^{2} \mathrm{~d} x= & -\int_{\Omega}\left[\frac{3}{2} u_{x}\left(\rho_{x}\right)^{2}+\rho \rho_{x} u_{x x}\right](t) \mathrm{d} x \\
& \leq C\left[\left|u_{x}\right|_{L^{\infty}}\left|\rho_{x}\right|_{L^{2}}^{2}+|\rho|_{L^{\infty}}\left|\rho_{x}\right|_{L^{2}}\left|u_{x x}\right|_{L^{2}}\right] \\
& \leq C|\rho|_{H^{1}}^{2}\left|u_{x x}\right|_{L^{2}} .
\end{aligned}
$$

From (2.10)and (2.11), by Gronwall's inequality, it follows that

$$
\sup _{0 \leq t \leq T}|\rho(t)|_{H^{1}}^{2} \leq\left|\rho_{0}\right|_{H^{1}}^{2} \exp \left\{C \int_{0}^{t}\left|u_{x x}\right|_{L^{2}} \mathrm{~d} s\right\} \leq C \exp \left(C \int_{0}^{t} \Psi^{\frac{6}{3 p-4} \gamma}(s) \mathrm{d} s\right),
$$

we can also get the following estimates. Using $(1.1)_{1}$ we obtain

$$
\left|\rho_{t}(t)\right|_{L^{2}} \leq\left|\rho_{x}(t)\right|_{L^{2}}|u(t)|_{L^{\infty}}+|\rho(t)|_{L^{\infty}}\left|u_{x}(t)\right|_{L^{2}} \leq C \Psi^{2}(t),
$$

where $C$ is a positive constant, depending only on $M_{0}$.

\subsection{Estimate for $\left|\eta_{t}\right|_{L^{2}}$ and $|\eta|_{H^{1}}$}

Multiplying $(1.1)_{3}$ by $\eta$, integrating the resulting equation over $\Omega_{T}$, using the boundary conditions (1.3) and Young's inequality, we have

$$
\begin{aligned}
& \int_{0}^{t}\left|\eta_{x}(s)\right|_{L^{2}}^{2} \mathrm{~d} s+\frac{1}{2}|\eta(t)|_{L^{2}}^{2} \\
& \quad \leq \iint_{\Omega_{T}}\left(\left|\eta u \eta_{x}\right|+\left|\eta \Phi_{x} \eta_{x}\right|\right) \mathrm{d} x \mathrm{~d} s \\
& \quad \leq \frac{1}{4} \int_{0}^{t}\left|\eta_{x}(s)\right|_{L^{2}}^{2} \mathrm{~d} s+C \int_{0}^{t}\left|u_{x}\right|_{L^{p}}^{2}|\eta|_{H^{1}}^{2} \mathrm{~d} s+C \int_{0}^{t}|\eta|_{H^{1}}^{2}+C \\
& \quad \leq \frac{1}{4} \int_{0}^{t}\left|\eta_{x}(s)\right|_{L^{2}}^{2} \mathrm{~d} s+C\left(1+\int_{0}^{t} \Psi^{4}(t) \mathrm{d} s\right)
\end{aligned}
$$


Multiplying $(1.1)_{3}$ by $\eta_{t}$, integrating (by parts) over $\Omega_{T}$, using the boundary conditions (1.3) and Young's inequality, we have

$$
\begin{aligned}
& \int_{0}^{t}\left|\eta_{t}(s)\right|_{L^{2}}^{2} \mathrm{~d} s+\frac{1}{2}\left|\eta_{x}(t)\right|_{L^{2}}^{2} \\
& \quad \leq \iint_{\Omega_{T}}\left|\eta\left(u-\Phi_{x}\right) \eta_{x t}\right| \mathrm{d} x \mathrm{~d} s \\
& \quad \leq \frac{1}{4} \int_{0}^{t}\left|\eta_{x t}(s)\right|_{L^{2}}^{2} \mathrm{~d} s+C \int_{0}^{t}|\eta|_{H^{1}}^{2}\left|u_{x}\right|_{L^{p}}^{2} \mathrm{~d} s+C \int_{0}^{t}|\eta|_{H^{1}}^{2} \mathrm{~d} s+C \\
& \quad \leq \frac{1}{4} \int_{0}^{t}\left|\eta_{x t}(s)\right|_{L^{2}}^{2} \mathrm{~d} s+C\left(1+\int_{0}^{t} \Psi^{4}(t) \mathrm{d} s\right) .
\end{aligned}
$$

Differentiating $(1.1)_{3}$ with respect to $t$, multiplying the resulting equation by $\eta_{t}$, integrating (by parts) over $\Omega_{T}$, we get

$$
\begin{aligned}
& \int_{0}^{t}\left|\eta_{x t}(s)\right|_{L^{2}}^{2} \mathrm{~d} s+\frac{1}{2}\left|\eta_{t}(t)\right|_{L^{2}}^{2} \\
& =\iint_{\Omega_{T}}\left(\eta\left(u-\Phi_{x}\right)\right)_{t} \eta_{x t} \mathrm{~d} x \mathrm{~d} s \\
& \leq C+\iint_{\Omega_{T}}\left(\left|\eta_{t} u \eta_{x t}\right|+\left|\eta_{t} \Phi_{x} \eta_{x t}\right|+\left|\eta_{x} u_{t} \eta_{t}\right|+\left|\eta u_{x t} \eta_{t}\right|\right) \mathrm{d} x \mathrm{~d} s \\
& \leq C\left(1+\int_{0}^{t}\left(\left|\eta_{t}\right|_{L^{2}}^{2}\left|u_{x}\right|_{L^{p}}^{2}+\left|\eta_{t}\right|_{L^{2}}^{2}+\left|\eta_{x}\right|_{L^{2}}^{2}\left|\eta_{t}\right|_{L^{2}}^{2}+|\eta|_{H^{1}}^{2}\left|\eta_{t}\right|_{L^{2}}^{2}\right) \mathrm{d} x\right) \\
& \quad+\frac{1}{2} \int_{0}^{t}\left|\eta_{x t}\right|_{L^{2}}^{2}+\frac{1}{2} \int_{0}^{t}\left|u_{x t}\right|_{L^{2}}^{2} \\
& \leq C\left(1+\int_{0}^{t} \Psi^{4}(s) \mathrm{d} s\right) .
\end{aligned}
$$

Combining (2.14)-(2.16) and (2.28), we get

$$
|\eta|_{H^{1}}^{2}+\left|\eta_{t}\right|_{L^{2}}^{2}+\int_{0}^{t}\left(\left|\eta_{x}\right|_{L^{2}}^{2}+\left|\eta_{t}\right|_{L^{2}}^{2}+\left|\eta_{x t}\right|_{L^{2}}^{2}\right)(s) \mathrm{d} s \leq C\left(1+\int_{0}^{t} \Psi^{4}(s) \mathrm{d} s\right) .
$$

\subsection{Estimate for $|u|_{w_{0}^{1, p}}$}

Using (2.1), we rewrite (2.1) as

$$
\rho u_{t}+\rho u u_{x}-\left[\left(\frac{\varepsilon u_{x}^{2}+1}{u_{x}^{2}+\varepsilon}\right)^{\frac{2-p}{2}} u_{x}\right]_{x}+(P+\eta)_{x}=-(\eta+\beta \rho) \Phi_{x} .
$$

Multiplying (2.18) by $u_{t}$, integrating (by parts) over $\Omega_{T}$, we have

$$
\begin{gathered}
\iint_{\Omega_{T}} \rho\left|u_{t}\right|^{2} \mathrm{~d} x \mathrm{~d} s+\iint_{\Omega_{T}}\left(\frac{\varepsilon u_{x}^{2}+1}{u_{x}^{2}+\varepsilon}\right)^{\frac{2-p}{2}} u_{x} u_{x t} \mathrm{~d} x \mathrm{~d} s \\
=-\iint_{\Omega_{T}}\left(\rho u u_{x}+P_{x}+\eta_{x}+(\eta+\beta \rho) \Phi_{x}\right) u_{t} \mathrm{~d} x \mathrm{~d} s .
\end{gathered}
$$


We deal with each term as follows:

$$
\begin{aligned}
& \int_{\Omega}\left(\frac{\varepsilon u_{x}^{2}+1}{u_{x}^{2}+\varepsilon}\right)^{\frac{2-p}{2}} u_{x} u_{x t} \mathrm{~d} x=\frac{1}{2} \int_{\Omega}\left(\frac{\varepsilon u_{x}^{2}+1}{u_{x}^{2}+\varepsilon}\right)^{\frac{2-p}{2}}\left(u_{x}^{2}\right)_{t} \mathrm{~d} x \\
& =\frac{1}{2} \frac{\mathrm{d}}{\mathrm{d} t} \int_{\Omega}\left(\int_{0}^{u_{x}^{2}}\left(\frac{\varepsilon s+1}{s+\varepsilon}\right)^{\frac{2-p}{2}} \mathrm{~d} s\right) \mathrm{d} x, \\
& \int_{0}^{u_{x}^{2}}\left(\frac{\varepsilon s+1}{s+\varepsilon}\right)^{\frac{2-p}{2}} \mathrm{~d} s \geq \int_{0}^{u_{x}^{2}}(s+1)^{\frac{2-p}{2}} \mathrm{~d} s=\frac{2}{p}\left[\left(u_{x}^{2}+1\right)^{\frac{p}{2}}-1\right], \\
& -\iint_{\Omega_{T}} P_{x} u_{t} \mathrm{~d} x \mathrm{~d} s=\iint_{\Omega_{T}} P u_{x t} \mathrm{~d} x \mathrm{~d} s=\frac{\mathrm{d}}{\mathrm{d} t} \iint_{\Omega_{T}} P u_{x} \mathrm{~d} x \mathrm{~d} s-\iint_{\Omega_{T}} P_{t} u_{x} \mathrm{~d} x \mathrm{~d} s .
\end{aligned}
$$

From $(1.1)_{1}$ we have

$$
\begin{aligned}
& P_{t}=-\gamma P u_{x}-P_{x} u, \\
& -\iint_{\Omega_{T}} \eta \Phi_{x} u_{t} \mathrm{~d} x \mathrm{~d} s=-\frac{\mathrm{d}}{\mathrm{d} t} \iint_{\Omega_{T}} \eta \Phi_{x} u \mathrm{~d} x \mathrm{~d} s+\iint_{\Omega_{T}} \eta_{t} \Phi_{x} u \mathrm{~d} x \mathrm{~d} s .
\end{aligned}
$$

Substituting the above into (2.19), using the Sobolev inequality and Young's inequality, we obtain

$$
\begin{aligned}
\int_{0}^{t} \mid & \left.\sqrt{\rho} u_{t}(s)\right|_{L^{2}} ^{2} \mathrm{~d} s+\left|u_{x}(t)\right|_{L^{p}}^{p} \\
\leq & \iint_{\Omega_{T}}\left(\left|\rho u u_{x} u_{t}\right|+\left|\gamma P u_{x}^{2}\right|+\left|P_{x} u u_{x}\right|+\left|\eta_{t} u_{x}\right|+\left|\eta_{t} \Phi_{x} u\right|+\left|\beta \rho \Phi_{x} u_{t}\right|\right) \mathrm{d} x \mathrm{~d} s \\
& +\int_{\Omega}\left(\left|P u_{x}\right|+\left|\eta u_{x}\right|+\left|\eta \Phi_{x} u\right|\right) \mathrm{d} x+C \\
\leq & C+\int_{0}^{t}\left(|\rho|_{L^{2}}|u|_{L^{\infty}}\left|u_{x}\right|_{L^{p}}^{\frac{p}{2}}\left|u_{x}\right|_{L^{\infty}}^{1-\frac{p}{2}}\left|u_{t}\right|_{L^{\infty}}+\gamma|P|_{L^{2}}\left|u_{x}\right|_{L^{p}}^{\frac{p}{2}}\left|u_{x}\right|_{L^{\infty}}^{1-\frac{p}{2}}\left|u_{x x}\right|_{L^{2}}\right. \\
& +a \gamma|\rho|_{L^{\infty}}^{\gamma-1}\left|\rho_{x}\right|_{L^{2}}\left|u_{x}\right|_{L^{p}}\left|u_{x x}\right|_{L^{2}}+\left|\eta_{t}\right|_{L^{2}}\left|u_{x}\right|_{L^{p}}^{\frac{p}{2}}\left|u_{x}\right|_{L^{\infty}}^{1-\frac{p}{2}}+\left|\eta_{t}\right|_{L^{2}}\left|\Phi_{x}\right|_{L^{2}}|u|_{L^{\infty}} \\
& \left.+\beta|\rho|_{L^{\infty}}^{\frac{1}{2}}\left|\Phi_{x}\right|_{L^{2}}\left|\sqrt{\rho} u_{t}\right|_{L^{2}}\right) \mathrm{d} s+|P|_{L^{\frac{p}{p-1}}}\left|u_{x}\right|_{L^{p}}+|\eta|_{L^{\frac{p}{p-1}}}\left|u_{x}\right|_{L^{p}}+|\eta|_{L^{\frac{p}{p-1}}}\left|\Phi_{x}\right|_{L^{p}}|u|_{L^{\infty}} \\
\leq & C\left(1+\int_{0}^{t}\left(|\rho|_{L^{2}}^{2}\left|u_{x}\right|_{L^{p}}^{2+p}\left|u_{x x}\right|_{L^{2}}^{2-p}+|P|_{L^{\infty}}\left|u_{x}\right|_{L^{p}}^{\frac{p}{2}}\left|u_{x x}\right|_{L^{2}}^{2-\frac{p}{2}}\right.\right. \\
& \left.\left.+|\rho|_{L^{\infty}}^{\gamma-1}\left|\rho_{x}\right|_{L^{2}}\left|u_{x}\right|_{L^{p}}\left|u_{x x}\right|_{L^{2}}+\left|\eta_{t}\right|_{L^{2}}\left|u_{x}\right|_{L^{p}}^{\frac{p}{2}}\left|u_{x x}\right|_{L^{2}}^{1-\frac{p}{2}}+\left|\eta_{t}\right|_{L^{2}}\left|u_{x}\right|_{L^{p}}+|\rho|_{L^{\infty}}\right) \mathrm{d} s\right) \\
& +|P|^{\frac{p}{p-1}}+|\eta|_{L^{p-1}}^{\frac{p}{p-1}}+\frac{1}{L^{p-1}} \int_{0}^{t}\left|\sqrt{\rho} u_{t}(s)\right|_{L^{2}}^{2} \mathrm{~d} s+\frac{1}{2}\left|u_{x}(t)\right|_{L^{p}}^{p} .
\end{aligned}
$$

To estimate (2.21), combining (2.20) we have the following estimates:

$$
\begin{aligned}
\int_{\Omega}|P(t)|^{\frac{p}{p-1}} \mathrm{~d} x & =\int_{\Omega}|P(0)|^{\frac{p}{p-1}} \mathrm{~d} x+\int_{0}^{t} \frac{\partial}{\partial s}\left(\int_{\Omega} P(s)^{\frac{p}{p-1}} \mathrm{~d} x\right) \mathrm{d} s \\
& \leq \int_{\Omega}|P(0)|^{\frac{p}{p-1}} \mathrm{~d} x+\frac{p}{p-1} \int_{0}^{t} \int_{\Omega} a \gamma \rho^{\gamma-1} P(s)^{\frac{p}{p-1}}\left(-\rho_{x} u-\rho u_{x}\right) \mathrm{d} x \mathrm{~d} s
\end{aligned}
$$




$$
\begin{aligned}
& \leq C+C \int_{0}^{t}|\rho|_{L^{\infty}}^{\gamma-1}|P|_{L^{\infty}}^{\frac{p}{p-1}}|\rho|_{H^{1}}\left|u_{x}\right|_{L^{p}} \mathrm{~d} s \\
& \leq C\left(1+\int_{0}^{t} \Psi^{\frac{1}{p-1}+\gamma+1}(s) \mathrm{d} s\right),
\end{aligned}
$$

following the same method, we get

$$
\int_{\Omega}|\eta(t)|^{\frac{p}{p-1}} \mathrm{~d} x \leq C\left(1+\int_{0}^{t} \Psi^{\frac{1}{p-1}+1}(s) \mathrm{d} s\right) .
$$

Combining (2.21)-(2.23) yields

$$
\int_{0}^{t}\left|\sqrt{\rho} u_{t}(s)\right|_{L^{2}}^{2}(s) \mathrm{d} s+\left|u_{x}(t)\right|_{L^{p}}^{p} \leq C\left(1+\int_{0}^{t} \Psi^{\frac{18 \gamma}{3 p-4}}(s) \mathrm{d} s\right),
$$

where $C$ is a positive constant, depending only on $M_{0}$.

\subsection{Estimate for $\left|\sqrt{\rho} u_{t}\right|_{L^{2}}$}

Differentiating $(1.1)_{2}$ with respect to $t$, we get

$$
\begin{gathered}
\rho u_{t t}+\rho u u_{x t}+\rho_{t} u_{t}+\rho_{t} u u_{x}+\rho u_{t} u_{x}+(P+\eta)_{x t} \\
=\left[\left(\frac{\varepsilon u_{x}^{2}+1}{u_{x}^{2}+\varepsilon}\right)^{\frac{2-p}{2}} u_{x}\right]_{x t}-\left(\eta_{t}+\beta \rho_{t}\right) \Phi_{x} .
\end{gathered}
$$

Multiplying the result equation by $u_{t}$, integrating over $\Omega$, we derive

$$
\begin{aligned}
& \frac{1}{2} \frac{\mathrm{d}}{\mathrm{d} t} \int_{\Omega} \rho\left|u_{t}\right|^{2} \mathrm{~d} x+\int_{\Omega}\left[\left(\frac{\varepsilon u_{x}^{2}+1}{u_{x}^{2}+\varepsilon}\right)^{\frac{2-p}{2}} u_{x}\right]_{t} u_{x t} \mathrm{~d} x \\
& \quad=\int_{\Omega}\left[(\rho u)_{x}\left(u_{t}^{2}+u u_{x} u_{t}\right)-(P+\eta)_{t} u_{x t}-\left(\eta_{t}+\beta \rho_{t}\right) \Phi_{x} u_{t}\right] \mathrm{d} x .
\end{aligned}
$$

Note that

$$
\begin{aligned}
& \int_{\Omega}\left[\left(\frac{\varepsilon u_{x}^{2}+1}{u_{x}^{2}+\varepsilon}\right)^{\frac{2-p}{2}} u_{x}\right]_{t} u_{x t} \mathrm{~d} x \\
& \quad=\int_{\Omega}\left[\left(\frac{\varepsilon u_{x}^{2}+1}{u_{x}^{2}+\varepsilon}\right)^{-\frac{p}{2}} u_{x}\right] \frac{\left(\varepsilon u_{x}^{2}+1\right)\left(u_{x}^{2}+\varepsilon\right)-(2-p)\left(1-\varepsilon^{2}\right) u_{x}^{2}}{\left(u_{x}^{2}+\varepsilon\right)^{2}} u_{x t}^{2} \mathrm{~d} x \\
& \quad \geq(p-1) \int_{\Omega}\left(u_{x}^{2}+1\right)^{\frac{p-2}{2}}\left|u_{x t}\right|^{2} \mathrm{~d} x .
\end{aligned}
$$

Let

$$
\zeta=\left(u_{x}^{2}+1\right)^{\frac{p-2}{4}},
$$

from (2.8), it follows that

$$
\left|\zeta^{-1}\right|_{L^{\infty}}=\left|\left(u_{x}^{2}+1\right)^{\frac{2-p}{4}}\right|_{L^{\infty}} \leq C\left(\left|u_{x x}\right|_{L^{2}}^{\frac{2-p}{2}}+1\right) \leq C \Psi^{\frac{3 \gamma}{3 p-4}}(t) .
$$


Combining (2.20), (2.25) can be rewritten as

$$
\begin{aligned}
& \frac{\mathrm{d}}{\mathrm{d} t} \int_{\Omega} \rho\left|u_{t}\right|^{2} \mathrm{~d} x+\int_{\Omega}\left|u_{x t}\right|^{2} \mathrm{~d} x \\
& \leq 2 \int_{\Omega} \rho|u|\left|u_{t}\right|\left|u_{x t}\right| \mathrm{d} x+\int_{\Omega}\left|\rho_{x}\right||u|^{2}\left|u_{x}\right|\left|u_{t}\right| \mathrm{d} x+\int_{\Omega} \rho|u|\left|u_{x}\right|^{2}\left|u_{t}\right| \mathrm{d} x \\
& \quad+\int_{\Omega} \gamma P\left|u_{x}\right|\left|u_{x t}\right| \mathrm{d} x+\int_{\Omega}\left|P_{x}\right||u|\left|u_{x t}\right| \mathrm{d} x+\int_{\Omega}\left|\eta_{t}\right|\left|\Phi_{x}\right|\left|u_{t}\right| \mathrm{d} x \\
& \quad+\int_{\Omega}\left|\beta \rho_{x}\right||u|\left|\Phi_{x}\right|\left|u_{t}\right| \mathrm{d} x+\int_{\Omega}\left|\beta \rho \| u_{x}\right|\left|\Phi_{x}\right|\left|u_{t}\right| \mathrm{d} x=\sum_{j=1}^{8} I_{j} .
\end{aligned}
$$

Using the Sobolev inequality, Young's inequality, $(1.1)_{2},(2.8)$, and (2.9), we obtain

$$
\begin{aligned}
I_{1} & \leq 2|\rho|_{L^{\infty}}^{\frac{1}{2}}|u|_{L^{\infty}}\left|\sqrt{\rho} u_{t}\right|_{L^{2}}\left|\zeta u_{x t}\right|_{L^{2}}\left|\zeta^{-1}\right|_{L^{\infty}} \leq C \Psi^{\frac{8 \gamma}{3 p-4}}(t)+\frac{1}{8}\left|u_{x t}\right|_{L^{2}}^{2} \\
I_{2} & \leq\left|\rho_{x}\right|_{L^{2}}|u|_{L^{\infty}}^{2}\left|u_{x}\right|_{L^{p}}\left|u_{x}\right|_{L^{\infty}}^{1-\frac{p}{2}}\left|u_{t}\right|_{L^{\infty}} \leq\left|\rho_{x}\right|_{L^{2}}\left|u_{x}\right|_{L^{p}}^{3}\left|u_{x x}\right|_{L^{2}}^{1-\frac{p}{2}}\left|\zeta u_{x t}\right|_{L^{2}}\left|\zeta^{-1}\right|_{L^{\infty}} \\
& \leq C \Psi^{\frac{12 \gamma}{3 p-4}}(t)+\frac{1}{8}\left|u_{x x}\right|_{L^{2}}^{2}, \\
I_{3} & \leq|\rho|_{L^{\frac{p}{p-1}}}|u|_{L^{\infty}}\left|u_{x}\right|_{L^{p}}\left|u_{x}\right|_{L^{\infty}}\left|u_{t}\right|_{L^{\infty}} \leq|\rho|_{L^{\infty}}\left|u_{x}\right|_{L^{p}}^{2}\left|u_{x x}\right|_{L^{2}}\left|\zeta u_{x t}\right|_{L^{2}}\left|\zeta^{-1}\right|_{L^{\infty}} \\
& \leq C \Psi^{\frac{15 \gamma}{3 p-4}}(t)+\frac{1}{8}\left|u_{x t}\right|_{L^{2}}^{2}, \\
I_{4} & \leq C|P|_{L^{2}}\left|u_{x}\right|_{L^{\infty}}\left|\zeta u_{x t}\right|_{L^{2}}\left|\zeta^{-1}\right|_{L^{\infty}} \leq C \Psi^{\frac{7 \gamma}{3 p-4}}(t)+\frac{1}{8}\left|u_{x t}\right|_{L^{2}}^{2}, \\
I_{5} & \leq\left|P_{x}\right|_{L^{2}}|u|_{L^{\infty}}\left|\zeta u_{x t}\right|_{L^{2}}\left|\zeta^{-1}\right|_{L^{\infty}} \leq C \Psi^{\frac{7 \gamma}{3 p-4}}(t)+\frac{1}{8}\left|u_{x t}\right|_{L^{2}}^{2} \\
I_{6} & \leq\left|\eta_{t}\right|_{L^{2}}\left|\Phi_{x}\right|_{L^{2}}\left|u_{t}\right|_{L^{\infty}} \leq C\left|\eta_{t}\right|_{L^{2}}\left|\zeta u_{x t}\right|_{L^{2}}\left|\zeta^{-1}\right|_{L^{\infty}} \leq C \Psi^{\frac{11 \gamma}{3 p-4}}(t)+\frac{1}{8}\left|u_{x t}\right|_{L^{2}}^{2} \\
I_{7} & \leq \beta\left|\rho_{x}\right|_{L^{2}}|u|_{L^{\infty}}\left|\Phi_{x}\right|_{L^{2}}\left|u_{t}\right|_{L^{\infty}} \leq C\left|\rho_{x}\right|_{L^{2}}\left|u_{x}\right|_{L^{p}}\left|\zeta u_{x t}\right|_{L^{2}}\left|\zeta^{-1}\right|_{L^{\infty}} \\
& \leq C \Psi^{\frac{9 \gamma}{3 p-4}}(t)+\frac{1}{8}\left|u_{x x}\right|_{L^{2}}^{2}, \\
I_{8} & \leq\left.\beta|\rho|_{L^{2}}\left|u_{x}\right|\right|_{L^{\infty}}\left|\Phi_{x}\right|_{L^{2}}\left|u_{t}\right|_{L^{\infty}} \leq C|\rho|_{L^{2}}\left|u_{x x}\right|_{L^{2}}\left|\zeta u_{x t}\right|_{L^{2}}\left|\zeta^{-1}\right|_{L^{\infty}} \\
& \leq C \Psi^{\frac{11 \gamma}{3 p-4}}(t)+\frac{1}{8}\left|u_{x t}\right|_{L^{2}}^{2} .
\end{aligned}
$$

Substituting $I_{j}(j=1,2, \ldots, 8)$ into (2.26), and integrating over $(\tau, t) \subset(0, T)$ over the time variable, we have

$$
\left|\sqrt{\rho} u_{t}(t)\right|_{L^{2}}^{2}+\int_{0}^{t}\left|u_{x t}\right|_{L^{2}}^{2}(s) \mathrm{d} s \leq\left|\sqrt{\rho} u_{t}(\tau)\right|_{L^{2}}^{2}+\int_{0}^{t} \Psi^{\frac{15 \gamma}{3 p-4}}(s) \mathrm{d} s .
$$

To obtain the estimate of $\left|\sqrt{\rho} u_{t}(t)\right|_{L^{2}}^{2}$, we need to estimate $\lim _{\tau \rightarrow 0}\left|\sqrt{\rho} u_{t}(\tau)\right|_{L^{2}}^{2}$. Multiplying (2.18) by $u_{t}$ and integrating over $\Omega$, we get

$$
\begin{aligned}
& \int_{\Omega} \rho\left|u_{t}\right|^{2} \mathrm{~d} x \\
& \quad \leq 2 \int_{\Omega}\left(\rho|u|^{2}\left|u_{x}\right|^{2}+\beta^{2} \rho\left|\Phi_{x}\right|^{2}+\rho^{-1}\left|\left[\left(\frac{\varepsilon u_{x}^{2}+1}{u_{x}^{2}+\varepsilon}\right)^{\frac{2-p}{2}} u_{x}\right]_{x}+(P+\eta)_{x}+\eta \Phi_{x}\right|^{2}\right) \mathrm{d} x .
\end{aligned}
$$


According to the smoothness of $(\rho, u, \eta)$, we obtain

$$
\begin{aligned}
& \lim _{\tau \rightarrow 0} \int_{\Omega}\left(\rho|u|^{2}\left|u_{x}\right|^{2}+\beta^{2} \rho\left|\Phi_{x}\right|^{2}+\rho^{-1}\left|\left[\left(\frac{\varepsilon u_{x}^{2}+1}{u_{x}^{2}+\varepsilon}\right)^{\frac{2-p}{2}} u_{x}\right]_{x}+(P+\eta)_{x}+\eta \Phi_{x}\right|^{2}\right) \mathrm{d} x \\
& =\int_{\Omega}\left(\rho\left|u_{0}\right|^{2}\left|u_{0 x}\right|^{2}+\beta^{2} \rho_{0}\left|\Phi_{x}\right|^{2}\right. \\
& \left.\quad+\rho_{0}^{-1}\left|\left[\left(\frac{\varepsilon u_{0 x}^{2}+1}{u_{0 x}^{2}+\varepsilon}\right)^{\frac{2-p}{2}} u_{0 x}\right]_{x}+\left(P_{0}+\eta_{0}\right)_{x}+\eta_{0} \Phi_{x}\right|^{2}\right) \mathrm{d} x \\
& \leq\left|\rho_{0}\right|_{L^{\infty}}\left|u_{0}\right|_{L^{\infty}}^{2}\left|u_{0 x}\right|_{L^{2}}^{2}+\beta^{2}\left|\rho_{0}\right|_{L^{\infty}}\left|\Phi_{x}\right|^{2}+|g|_{L^{2}}^{2}+\beta\left|\Phi_{x}\right|_{L^{2}}^{2} \leq C .
\end{aligned}
$$

Therefore, taking the limit on $\tau$ in (2.27), as $\tau \rightarrow 0$, we conclude that

$$
\left|\sqrt{\rho} u_{t}(t)\right|_{L^{2}}^{2}+\int_{0}^{t}\left|u_{x t}\right|_{L^{2}}^{2}(s) \mathrm{d} s \leq C\left(1+\int_{0}^{t} \Psi^{\frac{15 \gamma}{3 p-4}}(s) \mathrm{d} s\right),
$$

where $C$ is a positive constant, depending only on $M_{0}$.

Combining the estimates of (2.8), (2.9), (2.12), (2.13), (2.17), (2.24), (2.28), and the definition of $\Psi(t)$, we conclude that

$$
\Psi(t) \leq C \exp \left(\tilde{C} \int_{0}^{t} \Psi^{\frac{18 \gamma}{3 p-4}}(s) \mathrm{d} s\right)
$$

where $C, \tilde{C}$ are positive constant, depending only on $M_{0}$. This means that there exist a time $T_{1}>0$ and a constant $C$, such that

$$
\begin{aligned}
& \text { ess } \sup _{0 \leq t \leq T_{1}}\left(|\rho|_{H^{1}}+|u|_{W_{0}^{1, p} \cap H^{2}}+|\eta|_{H^{2}}+\left|\eta_{t}\right|_{L^{2}}+\left|\sqrt{\rho} u_{t}\right|_{L^{2}}+\left|\rho_{t}\right|_{L^{2}}\right) \\
& +\int_{0}^{T_{1}}\left(\left|\sqrt{\rho} u_{t}\right|_{L^{2}}^{2}+\left|u_{x t}\right|_{L^{2}}^{2}+\left|\eta_{x}\right|_{L^{2}}^{2}+\left|\eta_{t}\right|_{L^{2}}^{2}+\left|\eta_{x t}\right|_{L^{2}}^{2}\right) \mathrm{d} s \leq C
\end{aligned}
$$

where $C$ is a positive constant, depending only on $M_{0}$.

\section{Proof of the main theorem}

In this section, the existence of strong solutions can be established by a standard argument, we construct the approximate solutions by using the iterative scheme, derive uniform bounds and thus obtain solutions of the original problem by passing to the limit. Our proof will be based on the usual iteration argument and some ideas developed in $[25,26]$. Precisely, we first define $u^{0}=0$ and assuming that $u^{k-1}$ was defined for $k \geq 1$, let $\rho^{k}, u^{k}, \eta^{k}$ be the unique smooth solution to the following problems:

$$
\begin{aligned}
& \rho_{t}^{k}+\rho_{x}^{k} u^{k-1}+\rho^{k} u_{x}^{k-1}=0, \\
& \rho^{k} u_{t}^{k}+\rho^{k} u^{k-1} u_{x}^{k}+L_{p} u^{k}+P_{x}^{k}+\eta_{x}^{k}=-\left(\eta^{k}+\beta \rho^{k}\right) \Phi_{x}, \\
& \eta_{t}^{k}+\left(\eta^{k}\left(u^{k-1}-\Phi_{x}\right)\right)_{x}=\eta_{x x}^{k},
\end{aligned}
$$

with the initial and boundary conditions

$$
\left.\left(\rho^{k}, u^{k}, \eta^{k}\right)\right|_{t=0}=\left(\rho_{0}, u_{0}, \eta_{0}\right)
$$




$$
\left.u^{k}\right|_{\partial \Omega}=\left.\left(\eta_{x}^{k}+\eta^{k} \Phi_{x}\right)\right|_{\partial \Omega}=0,
$$

where

$$
L_{p} u^{k}=-\left[\left(\frac{\varepsilon\left(u_{x}^{k}\right)^{2}+1}{\left(u_{x}^{k}\right)^{2}+\varepsilon}\right)^{\frac{2-p}{2}} u_{x}^{k}\right]_{x} .
$$

With the process, the nonlinear coupled system has been reduced to a sequence of decoupled problems and each problem admits a smooth solution. The following estimates hold:

$$
\begin{aligned}
& \operatorname{ess} \sup _{0 \leq t \leq T_{1}}\left(\left|\rho^{k}\right|_{H^{1}}+\left|u^{k}\right|_{W_{0}^{1, p} \cap H^{2}}+\left|\eta^{k}\right|_{H^{2}}+\left|\eta_{t}^{k}\right|_{L^{2}}+\left|\sqrt{\rho^{k}} u_{t}^{k}\right|_{L^{2}}+\left|\rho_{t}^{k}\right|_{L^{2}}\right) \\
& \quad+\int_{0}^{T_{1}}\left(\left|\sqrt{\rho^{k}} u_{t}^{k}\right|_{L^{2}}^{2}+\left|u_{x t}^{k}\right|_{L^{2}}^{2}+\left|\eta_{x}^{k}\right|_{L^{2}}^{2}+\left|\eta_{t}^{k}\right|_{L^{2}}^{2}+\left|\eta_{x t}^{k}\right|_{L^{2}}^{2}\right) \mathrm{d} s \leq C,
\end{aligned}
$$

where $C$ is a generic constant depending only on $M_{0}$, but independent of $k$.

In addition, we first find $\rho^{k}$ from the initial problem

$$
\rho_{t}^{k}+u^{k-1} \rho_{x}^{k}+u_{x}^{k-1} \rho^{k}=0 \quad \text { and }\left.\quad \rho^{k}\right|_{t=0}=\rho_{0}
$$

with smooth function $u^{k-1}$, obviously, there is a unique solution $\rho^{k}$ on the above problem and also by a standard argument, we obtain

$$
\rho^{k}(x, t) \geq \delta \exp \left[-\int_{0}^{T_{1}}\left|u_{x}^{k-1}(\cdot, s)\right|_{L^{\infty}} \mathrm{d} s\right]>0, \quad \text { for all } t \in\left(0, T_{1}\right) .
$$

Next, we will prove that the approximate solution $\left(\rho^{k}, u^{k}, \eta^{k}\right)$ converges to a limit $\left(\rho^{\varepsilon}, u^{\varepsilon}, \eta^{\varepsilon}\right)$ in a strong sense. To this end, let us define

$$
\bar{\rho}^{k+1}=\rho^{k+1}-\rho^{k}, \quad \bar{u}^{k+1}=u^{k+1}-u^{k}, \quad \bar{\eta}^{k+1}=\eta^{k+1}-\eta^{k},
$$

then we easily verify that the functions $\bar{\rho}^{k+1}, \bar{u}^{k+1}, \bar{\eta}^{k+1}$ satisfy the system of equations

$$
\begin{aligned}
& \bar{\rho}_{t}^{k+1}+\left(\bar{\rho}^{k+1} u^{k}\right)_{x}+\left(\rho^{k} \bar{u}^{k}\right)_{x}=0 \\
& \rho^{k+1} \bar{u}_{t}^{k+1}+\rho^{k+1} u^{k} \bar{u}_{x}^{k+1}+\left(L_{p} u^{k+1}-L_{p} u^{k}\right) \\
& \quad=-\bar{\rho}^{k+1} u_{t}^{k}-\rho^{k} \bar{u}^{k} u_{x}^{k}-\bar{\rho}^{k+1} u^{k} u_{x}^{k}-\left(P_{x}^{k+1}-P_{x}^{k}\right)-\bar{\eta}_{x}^{k+1}-\left(\bar{\eta}^{k+1}+\beta \bar{\eta}^{k+1}\right) \Phi_{x} \\
& \bar{\eta}_{t}^{k+1}+\left(\eta^{k} \bar{u}^{k}\right)_{x}+\left(\bar{\eta}^{k+1}\left(u^{k}-\Phi_{x}\right)\right)_{x}=\bar{\eta}_{x x}^{k+1} .
\end{aligned}
$$

Multiplying (3.7) by $\bar{\rho}^{k+1}$, integrating over $\Omega$ and using Young's inequality, we obtain

$$
\begin{aligned}
\frac{\mathrm{d}}{\mathrm{d} t}\left|\bar{\rho}^{k+1}\right|_{L^{2}}^{2} & \leq C\left|\bar{\rho}^{k+1}\right|_{L^{2}}^{2}\left|u_{x}^{k}\right|_{L^{\infty}}+\left|\rho^{k}\right|_{H^{1}}\left|\bar{u}_{x}^{k}\right|_{L^{2}}\left|\bar{\rho}^{k+1}\right|_{L^{2}} \\
& \leq C\left|u_{x x}^{k}\right|_{L^{2}}\left|\bar{\rho}^{k+1}\right|_{L^{2}}^{2}+C_{\xi}\left|\rho^{k}\right|_{H^{1}}^{2}\left|\bar{\rho}^{k+1}\right|_{L^{2}}^{2}+\xi\left|\bar{u}_{x}^{k}\right|_{L^{2}}^{2} \\
& \leq C_{\xi}\left|\bar{\rho}^{k+1}\right|_{L^{2}}^{2}+\xi\left|\bar{u}_{x}^{k}\right|_{L^{2}}^{2},
\end{aligned}
$$

where $C_{\zeta}$ is a positive constant, depending on $M_{0}$ and $\zeta$ for all $t<T_{1}$ and $k \geq 1$. 
Multiplying (3.8) by $\bar{u}^{k+1}$, integrating over $\Omega$, and using Young's inequality, we obtain

$$
\begin{aligned}
& \frac{\mathrm{d}}{\mathrm{d} t} \int_{\Omega} \rho^{k+1}\left|\bar{u}^{k+1}\right|^{2} \mathrm{~d} x+\int_{\Omega}\left(L_{p} u^{k+1}-L_{p} u^{k}\right) \bar{u}^{k+1} \mathrm{~d} x \\
& \leq C \int_{\Omega}\left(\left|\bar{\rho}^{k+1}\right|\left(\left|u_{t}^{k}\right|+\left|u^{k} u_{x}^{k}\right|\right)\left|\bar{u}^{k+1}\right|+\rho^{k}\left|\bar{u}^{k}\right|\left|u_{x}^{k}\right|\left|\bar{u}^{k+1}\right|+\left|P^{k+1}-P^{k}\right|\left|\bar{u}_{x}^{k+1}\right|\right. \\
&\left.+\left|\bar{\eta}^{k+1}\right|\left|\bar{u}_{x}^{k+1}\right|+\left|\bar{\eta}^{k+1}+\beta \bar{\rho}^{k+1}\right|\left|\Phi_{x} \bar{u}^{k+1}\right|\right) \mathrm{d} x \\
& \leq C\left(\left|\bar{\rho}^{k+1}\right|_{L^{2}}\left|u_{x t}^{k}\right|_{L^{2}}\left|\bar{u}_{x}^{k+1}\right|_{L^{2}}+\left|\bar{\rho}^{k+1}\right|_{L^{2}}\left|u_{x}^{k}\right|_{L^{p}}\left|u_{x x}^{k}\right|_{L^{2}}\left|\bar{u}_{x}^{k+1}\right|_{L^{2}}\right. \\
&+\left|\rho^{k}\right|_{L^{2}}^{\frac{1}{2}}\left|\sqrt{\rho^{k}} \bar{u}^{k}\right|_{L^{2}}\left|u_{x x}^{k}\right|_{L^{2}}\left|\bar{u}_{x}^{k+1}\right|_{L^{2}}+\left|P^{k+1}-P^{k}\right|_{L^{2}}\left|\bar{u}_{x}^{k+1}\right|_{L^{2}} \\
&\left.+\left|\bar{\eta}^{k+1}\right|_{L^{2}}\left|\bar{u}_{x}^{k+1}\right|_{L^{2}}+\left|\bar{\rho}^{k+1}\right|_{L^{2}}\left|\bar{u}_{x}^{k+1}\right|_{L^{2}}+\left|\bar{\eta}^{k+1}\right|_{L^{2}}\left|\bar{u}_{x}^{k+1}\right|_{L^{2}}\right) .
\end{aligned}
$$

Let

$$
\sigma(s)=\left(\frac{\varepsilon s^{2}+1}{s^{2}+\varepsilon}\right)^{\frac{2-p}{2}} s
$$

then

$$
\sigma^{\prime}(s)=\left(\frac{\varepsilon s^{2}+1}{s^{2}+\varepsilon}\right)^{-\frac{p}{2}} \frac{\left(\varepsilon s^{2}+1\right)\left(s^{2}+\varepsilon\right)-(2-p)\left(1-\varepsilon^{2}\right) s^{2}}{\left(s^{2}+\varepsilon\right)^{2}} \geq \frac{p-1}{\left(s^{2}+\varepsilon\right)^{\frac{2-p}{2}}} .
$$

We estimate the second term of (3.11) as follows:

$$
\begin{aligned}
\int_{\Omega}\left(L_{p} u^{k+1}-L_{p} u^{k}\right) \bar{u}^{k+1} \mathrm{~d} x & =\int_{\Omega} \int_{0}^{1} \sigma^{\prime}\left(\theta u_{x}^{k+1}+(1-\theta) u_{x}^{k}\right) \mathrm{d} \theta\left|\bar{u}_{x}^{k+1}\right|^{2} \mathrm{~d} x \\
& \geq \int_{\Omega}\left[\int_{0}^{1} \frac{\mathrm{d} \theta}{\left|\theta u_{x}^{k+1}+(1-\theta) u_{x}^{k}\right|_{L^{\infty}}^{2-p}+1}\right]\left(\bar{u}_{x}^{k+1}\right)^{2} \\
& \geq C^{-1} \int_{\Omega}\left|\bar{u}_{x}^{k+1}\right|^{2} \mathrm{~d} x .
\end{aligned}
$$

Using (3.6), (3.12), and Young's inequality, (3.11) can be rewritten as

$$
\begin{aligned}
& \frac{\mathrm{d}}{\mathrm{d} t} \int_{\Omega} \rho^{k+1}\left|\bar{u}^{k+1}\right|^{2} \mathrm{~d} x+C^{-1} \int_{\Omega}\left|\bar{u}_{x}^{k+1}\right|^{2} \mathrm{~d} x \\
& \quad \leq B_{\xi}(t)\left|\bar{\rho}^{k+1}\right|_{L^{2}}^{2}+C\left(\left|\sqrt{\rho^{k}} \bar{u}^{k}\right|_{L^{2}}^{2}+\left|\bar{\eta}^{k+1}\right|_{L^{2}}^{2}\right)+\xi\left|\bar{u}_{x}^{k+1}\right|_{L^{2}}^{2},
\end{aligned}
$$

where $B_{\xi}(t)=C\left(1+\left|u_{x t}^{k}(t)\right|_{L^{2}}^{2}\right)$, for all $t \leq T_{1}$ and $k \geq 1$. Using (3.6) we derive

$$
\int_{0}^{t} B_{\xi}(s) \mathrm{d} s \leq C+C t
$$

Multiplying (3.9) by $\bar{\eta}^{k+1}$, integrating over $\Omega$, and using (3.6) and Young's inequality, we have

$$
\begin{aligned}
& \frac{1}{2} \frac{\mathrm{d}}{\mathrm{d} t} \int_{\Omega}\left|\bar{\eta}^{k+1}\right|^{2} \mathrm{~d} x+\int_{\Omega}\left|\bar{\eta}_{x}^{k+1}\right|^{2} \mathrm{~d} x \\
& \quad \leq \int_{\Omega}\left|\bar{\eta}^{k+1}\right|\left|u^{k}-\Phi_{x}\right|\left|\bar{\eta}_{x}^{k+1}\right| \mathrm{d} x+\int_{\Omega}\left(\left|\eta^{k}\right|\left|\bar{u}^{k}\right|\right)_{x}\left|\bar{\eta}^{k+1}\right| \mathrm{d} x
\end{aligned}
$$




$$
\begin{aligned}
& \leq\left|\bar{\eta}^{k+1}\right|_{L^{2}}\left|u^{k}-\Phi_{x}\right|_{L^{\infty}}\left|\bar{\eta}_{x}^{k+1}\right|_{L^{2}}+\left|\eta_{x}^{k}\right|_{L^{2}}\left|\bar{u}^{k}\right|_{L^{\infty}}\left|\bar{\eta}^{k+1}\right|_{L^{2}}+\left|\eta^{k}\right|_{L^{\infty}}\left|\bar{u}_{x}^{k}\right|_{L^{2}}\left|\bar{\eta}^{k+1}\right|_{L^{2}} \\
& \leq C_{\xi}\left|\bar{\eta}^{k+1}\right|_{L^{2}}^{2}+\xi\left|\bar{\eta}_{x}^{k+1}\right|_{L^{2}}^{2}+\xi\left|\bar{u}_{x}^{k}\right|_{L^{2}}^{2} .
\end{aligned}
$$

Collecting (3.10), (3.13), and (3.14), we obtain

$$
\begin{aligned}
& \frac{\mathrm{d}}{\mathrm{d} t}\left(\left|\bar{\rho}^{k+1}(t)\right|_{L^{2}}^{2}+\left|\sqrt{\rho^{k+1}} \bar{u}^{k+1}(t)\right|_{L^{2}}^{2}+\left|\bar{\eta}^{k+1}(t)\right|_{L^{2}}^{2}\right)+\left|\bar{u}_{x}^{k+1}(t)\right|_{L^{2}}^{2}+\left|\bar{\eta}_{x}^{k+1}\right|_{L^{2}}^{2} \\
& \quad \leq E_{\xi}(t)\left|\bar{\rho}^{k+1}(t)\right|_{L^{2}}^{2}+C\left|\sqrt{\rho^{k}} \bar{u}^{k}\right|_{L^{2}}^{2}+C_{\xi}\left|\bar{\eta}^{k+1}\right|_{L^{2}}^{2}+\xi\left|\bar{u}_{x}^{k}\right|_{L^{2}}^{2},
\end{aligned}
$$

with $E_{\zeta}(t)$ depending only on $B_{\zeta}(t)$ and $C_{\xi}$, for all $t \leq T_{1}$ and $k \geq 1$. Using (3.6), we have

$$
\int_{0}^{t} E_{\xi}(s) \mathrm{d} s \leq C+C_{\xi} t
$$

Integrating (3.15) over $(0, t) \subset\left(0, T_{1}\right)$ with respect to $t$, using Gronwall's inequality, we have

$$
\begin{aligned}
& \left|\bar{\rho}^{k+1}(t)\right|_{L^{2}}^{2}+\left|\sqrt{\rho^{k+1}} \bar{u}^{k+1}(t)\right|_{L^{2}}^{2}+\left|\bar{\eta}^{k+1}(t)\right|_{L^{2}}^{2}+\int_{0}^{t}\left|\bar{u}_{x}^{k+1}(t)\right|_{L^{2}}^{2} \mathrm{~d} s+\int_{0}^{t}\left|\bar{\eta}_{x}^{k+1}\right|_{L^{2}}^{2} \mathrm{~d} s \\
& \quad \leq C \exp \left(C_{\xi} t\right) \int_{0}^{t}\left(\left|\sqrt{\rho^{k}} \bar{u}^{k}(s)\right|_{L^{2}}^{2}+\left|\bar{u}_{x}^{k}(s)\right|_{L^{2}}^{2}\right) \mathrm{d} s .
\end{aligned}
$$

From the above recursive relation, choose $\xi>0$ and $0<T_{*}<T_{1}$ such that $C \exp \left(C_{\xi} T_{*}\right)<\frac{1}{2}$, using Gronwall's inequality, we deduce that

$$
\begin{aligned}
& \sum_{k=1}^{K}\left[\sup _{0 \leq t \leq T_{*}}\left(\left|\bar{\rho}^{k+1}(t)\right|_{L^{2}}^{2}+\left|\sqrt{\rho^{k+1}} \bar{u}^{k+1}(t)\right|_{L^{2}}^{2}+\left|\bar{\eta}^{k+1}(t)\right|_{L^{2}}^{2}\right)\right. \\
& \left.\quad+\int_{0}^{T_{*}}\left|\bar{u}_{x}^{k+1}(t)\right|_{L^{2}}^{2} \mathrm{~d} t+\int_{0}^{T_{*}}\left|\bar{\eta}_{x}^{k+1}(t)\right|_{L^{2}}^{2} \mathrm{~d} t\right]<C,
\end{aligned}
$$

where $C$ is a positive constant, depending only on $M_{0}$.

Therefore, as $k \rightarrow+\infty$, the sequence $\left(\rho^{k}, u^{k}, \eta^{k}\right)$ converges to a limit $\left(\rho^{\varepsilon}, u^{\varepsilon}, \eta^{\varepsilon}\right)$ in the following strong sense:

$$
\begin{aligned}
& \rho^{k} \rightarrow \rho^{\varepsilon} \quad \text { in } L^{\infty}\left(0, T_{*} ; L^{2}(\Omega)\right), \\
& u^{k} \rightarrow u^{\varepsilon} \quad \text { in } L^{\infty}\left(0, T_{*} ; L^{2}(\Omega)\right) \cap L^{2}\left(0, T_{*} ; H_{0}^{1}(\Omega)\right), \\
& \eta^{k} \rightarrow \eta^{\varepsilon} \quad \text { in } L^{\infty}\left(0, T_{*} ; L^{2}(\Omega)\right) \cap L^{2}\left(0, T_{*} ; H^{1}(\Omega)\right) .
\end{aligned}
$$

By virtue of the lower semi-continuity of various norms, we deduce from the uniform estimate (3.6) that $\left(\rho^{\varepsilon}, u^{\varepsilon}, \eta^{\varepsilon}\right)$ satisfies the following uniform estimate:

$$
\begin{aligned}
& \text { ess } \sup _{0 \leq t \leq T_{1}}\left(\left|\rho^{\varepsilon}\right|_{H^{1}}+\left|u^{\varepsilon}\right|_{W_{0}^{1, p} \cap H^{2}}+\left|\eta^{\varepsilon}\right|_{H^{2}}+\left|\eta_{t}^{\varepsilon}\right|_{L^{2}}+\left|\sqrt{\rho^{\varepsilon}} u_{t}^{\varepsilon}\right|_{L^{2}}+\left|\rho_{t}^{\varepsilon}\right|_{L^{2}}\right) \\
& \quad+\int_{0}^{T_{*}}\left(\left|\sqrt{\rho^{\varepsilon}} u_{t}^{\varepsilon}\right|_{L^{2}}^{2}+\left|u_{x t}^{\varepsilon}\right|_{L^{2}}^{2}+\left|\eta_{x}^{\varepsilon}\right|_{L^{2}}^{2}+\left|\eta_{t}^{\varepsilon}\right|_{L^{2}}^{2}+\left|\eta_{x t}^{\varepsilon}\right|_{L^{2}}^{2}\right) \mathrm{d} s \leq C
\end{aligned}
$$


Since all of the constants do not depend on $\varepsilon$, there exists a subsequence $\left(\rho^{\varepsilon_{j}}, u^{\varepsilon_{j}}, \eta^{\varepsilon_{j}}\right)$ of $\left(\rho^{\varepsilon}, u^{\varepsilon}, \eta^{\varepsilon}\right)$, that, without loss of generality, we denote $\left(\rho^{\varepsilon}, u^{\varepsilon}, \eta^{\varepsilon}\right)$. Let $\varepsilon \rightarrow 0$, then we obtain the following convergence:

$$
\begin{aligned}
& \rho^{\varepsilon} \rightarrow \rho^{\delta} \quad \text { in } L^{\infty}\left(0, T_{*} ; L^{2}(\Omega)\right), \\
& u^{\varepsilon} \rightarrow u^{\delta} \quad \text { in } L^{\infty}\left(0, T_{*} ; L^{2}(\Omega)\right) \cap L^{2}\left(0, T_{*} ; H_{0}^{1}(\Omega)\right), \\
& \eta^{\varepsilon} \rightarrow \eta^{\delta} \quad \text { in } L^{\infty}\left(0, T_{*} ; L^{2}(\Omega)\right) \cap L^{2}\left(0, T_{*} ; H^{1}(\Omega)\right),
\end{aligned}
$$

and also

$$
\begin{aligned}
& \text { ess } \sup _{0 \leq t \leq T_{1}}\left(\left|\rho^{\delta}\right|_{H^{1}}+\left|u^{\delta}\right|_{W_{0}^{1, p} \cap H^{2}}+\left|\eta^{\delta}\right|_{H^{2}}+\left|\eta_{t}^{\delta}\right|_{L^{2}}+\left|\sqrt{\rho^{\delta}} u_{t}^{\delta}\right|_{L^{2}}+\left|\rho_{t}^{\delta}\right|_{L^{2}}\right) \\
& +\int_{0}^{T_{*}}\left(\left|\sqrt{\rho^{\delta}} u_{t}^{\delta}\right|_{L^{2}}^{2}+\left|u_{x t}^{\delta}\right|_{L^{2}}^{2}+\left|\eta_{x}^{\delta}\right|_{L^{2}}^{2}+\left|\eta_{t}^{\delta}\right|_{L^{2}}^{2}+\left|\eta_{x t}^{\delta}\right|_{L^{2}}^{2}\right) \mathrm{d} s \leq C .
\end{aligned}
$$

For each small $\delta>0$, let $\rho_{0}^{\delta}=J_{\delta} * \rho_{0}+\delta$, $J_{\delta}$ is a mollifier on $\Omega$, and $u_{0}^{\delta} \in H_{0}^{1}(\Omega) \cap H^{2}(\Omega)$ is a smooth solution of the boundary value problem

$$
\left\{\begin{array}{l}
L_{p} u_{0}^{\delta}=\left(P\left(\rho_{0}^{\delta}\right)+\eta_{0}^{\delta}\right)_{x}+\eta_{0}^{\delta} \Phi_{x}+\rho_{0}^{\delta}\left(g^{\delta}+\beta \Phi_{x}\right), \\
u_{0}^{\delta}(0)=u_{0}^{\delta}(1)=0
\end{array}\right.
$$

where $g^{\delta} \in C_{0}^{\infty}$ and satisfies $\left|g^{\delta}\right|_{L^{2}} \leq|g|_{L^{2}}, \lim _{\delta \rightarrow 0^{+}}\left|g^{\delta}-g\right|_{L^{2}}=0$.

We deduce that $\left(\rho^{\delta}, u^{\delta}, \eta^{\delta}\right)$ is a solution of the following initial boundary value problem:

$$
\left\{\begin{array}{l}
\rho_{t}+(\rho u)_{x}=0, \\
(\rho u)_{t}+\left(\rho u^{2}\right)_{x}-\lambda\left(\left|u_{x}\right|^{p-2} u_{x}\right)_{x}+(P+\eta)_{x}=-(\eta+\beta \rho) \Phi_{x}, \\
\eta_{t}+\left(\eta\left(u-\Phi_{x}\right)\right)_{x}=\eta_{x x}, \\
\left.(\rho, u, \eta)\right|_{t=0}=\left(\rho_{0}^{\delta}, u_{0}^{\delta}, \eta_{0}^{\delta}\right), \\
\left.u\right|_{\partial \Omega}=\left.\left(\eta_{x}+\eta \Phi_{x}\right)\right|_{\partial \Omega}=0,
\end{array}\right.
$$

where $\rho_{0}^{\delta} \geq \delta, \frac{4}{3}<p<2$.

By the proof of Lemma 2.3 in [16], there exists a subsequence $\left\{u_{0}^{\delta_{j}}\right\}$ of $\left\{u_{0}^{\delta}\right\}$, as $\delta_{j} \rightarrow 0^{+}$, $u_{0}^{\delta} \rightarrow u_{0}$ in $H_{0}^{1}(\Omega) \cap H^{2}(\Omega),-\left(\left|u_{0 x}^{\delta_{j}}\right|^{p-2} u_{0 x}^{\delta_{j}}\right)_{x} \rightarrow-\left(\left|u_{0 x}\right|^{p-2} u_{0 x}\right)_{x}$ in $L^{2}(\Omega)$, Hence, $u_{0}$ satisfies the compatibility condition (1.5) of Theorem 1.1. By virtue of the lower semi-continuity of various norms, we deduce that $(\rho, u, \eta)$ satisfies the following uniform estimate:

$$
\begin{aligned}
& \operatorname{ess} \sup _{0 \leq t \leq T_{1}}\left(|\rho|_{H^{1}}+|u|_{W_{0}^{1, p} \cap H^{2}}+|\eta|_{H^{2}}+\left|\eta_{t}\right|_{L^{2}}+\left|\sqrt{\rho} u_{t}\right|_{L^{2}}+\left|\rho_{t}\right|_{L^{2}}\right) \\
& \quad+\int_{0}^{T_{*}}\left(\left|\sqrt{\rho} u_{t}\right|_{L^{2}}^{2}+\left|u_{x t}\right|_{L^{2}}^{2}+\left|\eta_{x}\right|_{L^{2}}^{2}+\left|\eta_{t}\right|_{L^{2}}^{2}+\left|\eta_{x t}\right|_{L^{2}}^{2}\right) \mathrm{d} s \leq C
\end{aligned}
$$

where $C$ is a positive constant, depending only on $M_{0}$. The uniqueness of the solution can also be obtained by the same method as the above proof of convergence, we omit the details here. This completes the proof. 


\section{Appendix}

In this section, for the sake of completeness, we give a slightly more particular result for the previous case.

Lemma A.1 Let $u_{0} \in H_{0}^{1}(\Omega) \cap H^{2}(\Omega), \rho_{0} \in H^{1}(\Omega), \eta_{0} \in H^{2}(\Omega), \Phi \in C^{2}(\Omega), g \in L^{2}(\Omega), u_{0}^{\varepsilon}$ is a solution of the boundary value problem

$$
\left\{\begin{array}{l}
{\left[\left(\frac{\varepsilon\left(u_{0 x}^{\varepsilon}\right)^{2}+1}{\left(u_{0 x}^{\varepsilon}\right)^{2}+\varepsilon}\right)^{\frac{2-p}{2}} u_{0 x}^{\varepsilon}\right]_{x}-\left(P\left(\rho_{0}\right)+\eta_{0}\right)_{x}-\eta_{0} \Phi_{x}=\rho_{0}\left(g+\beta \Phi_{x}\right)} \\
u_{0}^{\varepsilon}(0)=u_{0}^{\varepsilon}(1)=0
\end{array}\right.
$$

Then there are a subsequence $\left\{u_{0}^{\varepsilon_{j}}\right\}, j=1,2,3, \ldots$, of $\left\{u_{0}^{\varepsilon}\right\}$ and $u_{0} \in H_{0}^{1}(\Omega) \cap H^{2}(\Omega)$ such that, as $\varepsilon_{j} \rightarrow 0$,

$$
\begin{aligned}
& u_{0}^{\varepsilon_{j}} \rightarrow u_{0} \quad \text { in } H_{0}^{1}(\Omega) \cap H^{2}(\Omega), \\
& {\left[\left(\frac{\varepsilon_{j}\left(u_{0 x}^{\varepsilon_{j}}\right)^{2}+1}{\left(u_{0 x}^{\varepsilon_{j}}\right)^{2}+\varepsilon_{j}}\right)^{\frac{2-p}{2}} u_{0 x}^{\varepsilon_{j}}\right]_{x} \rightarrow\left(\left|u_{0 x}\right|^{p-2} u_{0 x}\right)_{x} \text { in } L^{2}(\Omega) .}
\end{aligned}
$$

Proof According to (A.1), we have

$$
u_{0 x x}^{\varepsilon}=\left(\frac{\varepsilon\left(u_{0 x}^{\varepsilon}\right)^{2}+1}{\left(u_{0 x}^{\varepsilon}\right)^{2}+\varepsilon}\right)^{\frac{p}{2}} \frac{\left(\left(u_{0 x}^{\varepsilon}\right)^{2}+\varepsilon\right)^{2}\left(\left(P\left(\rho_{0}\right)+\eta_{0}\right)_{x}+\eta_{0} \Phi_{x}+\rho_{0}\left(g+\beta \Phi_{x}\right)\right)}{\left(\varepsilon\left(u_{0 x}^{\varepsilon}\right)^{2}+1\right)\left(\left(u_{0 x}^{\varepsilon}\right)^{2}+\varepsilon\right)-(2-p)\left(1-\varepsilon^{2}\right)\left(u_{0 x}^{\varepsilon}\right)^{2}} .
$$

Then

$$
\begin{aligned}
\left|u_{0 x x}^{\varepsilon}\right|_{L^{2}} & \leq\left|\left(\frac{\varepsilon\left(u_{0 x}^{\varepsilon}\right)^{2}+1}{\left(u_{0 x}^{\varepsilon}\right)^{2}+\varepsilon}\right)^{1-\frac{p}{2}}\right|_{L^{\infty}}\left|\left(P\left(\rho_{0}\right)+\eta_{0}\right)_{x}+\eta_{0} \Phi_{x}+\rho_{0}\left(g+\beta \Phi_{x}\right)\right|_{L^{2}} \\
& \leq\left(\left|u_{0 x}^{\varepsilon}\right|_{L^{\infty}}^{2}+1\right)^{1-\frac{p}{2}}\left|\left(P\left(\rho_{0}\right)+\eta_{0}\right)_{x}+\eta_{0} \Phi_{x}+\rho_{0}\left(g+\beta \Phi_{x}\right)\right|_{L^{2}},
\end{aligned}
$$

then we get

$$
\left|u_{0 x x}^{\varepsilon}\right|_{L^{2}} \leq C\left(1+\left|\left(P\left(\rho_{0}\right)+\eta_{0}\right)_{x}+\eta_{0} \Phi_{x}+\rho_{0}\left(g+\beta \Phi_{x}\right)\right|_{L^{2}}\right)^{\frac{1}{p-1}} \leq C .
$$

Therefore, by the above inequality, as $\varepsilon_{j} \rightarrow 0$,

$$
\begin{aligned}
& u_{0}^{\varepsilon_{j}} \rightarrow u_{0} \quad \text { in } C^{\frac{3}{2}}(\Omega), \\
& u_{0 x x}^{\varepsilon_{j}} \rightarrow u_{0 x x} \quad \text { in } L^{2}(\Omega) \text { weakly. }
\end{aligned}
$$

Thus, we see that $\left\{u_{0 x}^{\varepsilon_{j}}\right\}$ is a Cauchy subsequence of $C^{\frac{3}{2}}(\Omega)$, for all $\alpha_{1}>0$, we find $N$, as $i, j>N$, we have

$$
\left|u_{0 x}^{\varepsilon_{i}}-u_{0 x}^{\varepsilon_{j}}\right|_{L^{\infty}(\Omega)}<\alpha_{1}
$$

Now, we prove that $\left\{u_{0 x x}^{\varepsilon}\right\}$ has a Cauchy sequence in $L_{2}$ norm. 
Let

$$
\phi_{i}=\phi\left(\left(u_{0 x}^{\varepsilon_{i}}\right)^{2}\right)=\left(\frac{\varepsilon_{i}\left(u_{0 x}^{\varepsilon}\right)^{2}+1}{\left(u_{0 x}^{\varepsilon_{i}}\right)^{2}+\varepsilon_{i}}\right)^{\frac{p}{2}} \frac{\left(\left(u_{0 x}^{\varepsilon_{i}}\right)^{2}+\varepsilon_{i}\right)^{2}}{\left(\varepsilon_{i}\left(u_{0 x}^{\varepsilon_{i}}\right)^{2}+1\right)\left(\left(u_{0 x}^{\varepsilon_{i}}\right)^{2}+\varepsilon_{i}\right)-(2-p)\left(1-\varepsilon_{i}^{2}\right)\left(u_{0 x}^{\varepsilon_{i}}\right)^{2}} .
$$

For all $\alpha>0$, there exists $N$, as $i, j>N$, we can deduce that

$$
\left|u_{0 x x}^{\varepsilon_{i}}-u_{0 x x}^{\varepsilon_{j}}\right|_{L^{2}(\Omega)} \leq\left|\phi_{i}-\phi_{j}\right|_{L^{\infty}(\Omega)}\left|\left(P\left(\rho_{0}\right)+\eta_{0}\right)_{x}+\eta_{0} \Phi_{x}+\rho_{0}\left(g+\beta \Phi_{x}\right)\right|_{L^{2}(\Omega)} .
$$

With the assumption, we obtain

$$
\left|\left(P\left(\rho_{0}\right)+\eta_{0}\right)_{x}+\eta_{0} \Phi_{x}+\rho_{0}\left(g+\beta \Phi_{x}\right)\right|_{L^{2}(\Omega)} \leq C,
$$

where $C$ is a positive constant, depending only on $\left|\rho_{0}\right|_{H^{1}(\Omega)},|g|_{L^{2}(\Omega)}$, and $\left|\eta_{0}\right|_{H^{2}(\Omega)}$. Use the following inequality:

$$
\left|\phi_{i}-\phi_{j}\right|_{L^{\infty}(\Omega)} \leq\left|\int_{0}^{1} \phi^{\prime}\left(\theta\left(u_{0 x}^{\varepsilon_{i}}\right)^{2}+(1-\theta)\left(u_{0 x}^{\varepsilon_{j}}\right)^{2}\right) d \theta\left(\left(u_{0 x}^{\varepsilon_{i}}\right)^{2}-\left(u_{0 x}^{\varepsilon_{j}}\right)^{2}\right)\right|_{L^{\infty}(\Omega)},
$$

where $0<\theta<1$.

By a simple calculation, we can get $\phi^{\prime}(s) \leq \frac{2}{p-1}\left(1+s^{-\frac{p}{2}}\right)$, where $C$ depends only on $p$, then

$$
\begin{aligned}
\left|\phi_{i}-\phi_{j}\right|_{L^{\infty}(\Omega)} \leq & \frac{2}{p-1}\left|\left(1+\int_{0}^{1}\left(\theta\left(u_{0 x}^{\varepsilon_{i}}\right)^{2}+(1-\theta)\left(u_{0 x}^{\varepsilon_{j}}\right)^{2}\right) d \theta\right)\left(\left(u_{0 x}^{\varepsilon_{i}}\right)^{2}-\left(u_{0 x}^{\varepsilon_{j}}\right)^{2}\right)\right|_{L^{\infty}(\Omega)} \\
\leq & \frac{2}{p-1}\left|u_{0 x}^{\varepsilon_{i}}-u_{0 x}^{\varepsilon_{j}}\right|_{L^{\infty}(\Omega)}\left|u_{0 x}^{\varepsilon_{i}}+u_{0 x}^{\varepsilon_{j}}\right|_{L^{\infty}(\Omega)} \\
& +\frac{4}{(2-p)(p-1)}\left|u_{0 x}^{\varepsilon_{i}}-u_{0 x}^{\varepsilon_{j}}\right|_{L^{\infty}(\Omega)}^{\frac{2-p}{2}}\left|u_{0 x}^{\varepsilon_{i}}+u_{0 x}^{\varepsilon_{j}}\right|_{L^{\infty}(\Omega)}^{\frac{2-p}{2}} \leq \alpha .
\end{aligned}
$$

Substituting this into (A.1), we have

$$
\left|u_{0 x x}^{\varepsilon_{i}}-u_{0 x x}^{\varepsilon_{j}}\right|_{L^{\infty}(\Omega)}<\alpha,
$$

then there is a subsequence $\left\{u_{0 x x}^{\varepsilon_{j}}\right\}$ and $\left\{u_{0 x x}^{\varepsilon}\right\}$, such that

$$
\left\{u_{0 x x}^{\varepsilon_{j}}\right\} \rightarrow \chi \quad \text { in } L^{2}(\Omega) .
$$

By the uniqueness of the weak convergence, we have

$$
\chi=\left\{u_{0 x x}^{\varepsilon}\right\} .
$$

Since $\left(P\left(\rho_{0}\right)+\eta\right)_{x}+\eta_{0} \Phi_{x}+\rho_{0}\left(g+\beta \Phi_{x}\right)$ are independent of $\varepsilon$, in the same way we obtain, as $\varepsilon_{j} \rightarrow 0$,

$$
\left[\left(\frac{\varepsilon_{j}\left(u_{0 x}^{\varepsilon_{j}}\right)^{2}+1}{\left(u_{0 x}^{\varepsilon_{j}}\right)^{2}+\varepsilon_{j}}\right)^{\frac{2-p}{2}} u_{0 x}^{\varepsilon_{j}}\right]_{x} \rightarrow\left(\left|u_{0 x}\right|^{p-2} u_{0 x}\right)_{x} \quad \text { in } L^{2}(\Omega) .
$$

This completes the proof of Lemma A.1. 


\section{Competing interests}

The authors declare that they have no competing interests.

\section{Authors' contributions}

All authors read and approved the final manuscript.

\section{Author details}

${ }^{1}$ College of Science, Liaoning University of Technology, Jinzhou, Liaoning 121001, P.R. China. ${ }^{2}$ Institute of Mathematics, Jilin University, Changchun, Jilin 130012, P.R. China.

\section{Acknowledgements}

The authors would like to thank an anonymous referee for his/her helpful comments and suggestions which improved the presentation of the paper. This work was supported by the Tian Yuan Mathematical Foundation of China (No. 11526105), the National Natural Science Foundation of China (No. 11572146), and the Scientific Research Foundation of Liaoning University of Technology (No. X201404).

Received: 13 March 2016 Accepted: 27 May 2016 Published online: 02 June 2016

\section{References}

1. Tory, EM, Karlsen, KH, Bürger, R, Berres, S: Strongly degenerate parabolic-hyperbolic systems modeling polydisperse sedimentation with compression. SIAM J. Appl. Math. 64, 41-80 (2003)

2. Baranger, C, Boudin, L, Jabin, PE, Mancini, S: A modeling of biospray for the upper airways. In: CEMRACS 2004 Mathematics and Applications to Biology and Medicine. ESAIM Proc., vol. 14, pp. 41-47 (2005)

3. Sartory, WK: Three-component analysis of blood sedimentation by the method of characteristics. Math. Biosci. 33, 145-165 (1977)

4. Spannenberg, A, Galvin, KP: Continuous differential sedimentation of a binary suspension. Chem. Eng. Aust. 21, 7-11 (1996)

5. Williams, FA: Combustion Theory, 2nd edn. Benjamin-Cummings, Redwood City (1985)

6. Williams, FA: Spray combustion and atomization. Phys. Fluids 1, 541-545 (1958)

7. Carrillo, JA, Karper, T, Trivisa, K: On the dynamics of a fluid-particle model: the bubbling regime. Nonlinear Anal., Real World Appl. 74, 2778-2801 (2011)

8. Lions, PL: Mathematical Topics in Fluid Dynamics. Volume 2: Compressible Models. Oxford University Press, Oxford (1998)

9. Feireisl, E, Petzeltová, H: Large time behavior of solutions to the Navier-Stokes equations of compressible flow. Arch. Ration. Mech. Anal. 150, 77-96 (1999)

10. Feireisl, E, Novotný, A, Petzeltová, H: On the existence of globally defined weak solution to the Navier-Stokes equations. J. Math. Fluid Mech. 3, 358-392 (2001)

11. Mellet, A, Vasseur, A: Asymptotic analysis for a Vlasov-Fokker-Planck/compressible Navier-Stokes system of equations. Commun. Math. Phys. 281, 573-596 (2008)

12. Chhabra, RP: Bubbles, Drops, and Particles in Non-Newtonian Fluids, 2nd edn. Taylor \& Francis, New York (2007)

13. Bellout, H, Bloom, F, Nečas, J: Existence, uniqueness and stability of solutions to the initial boundary value problem for bipolar viscous fluids. Differ. Integral Equ. 8, 453-464 (1994)

14. Guo, B, Zhu, P: Partial regularity of suitable weak solutions to the system of the incompressible non-Newtonian fluids. J. Differ. Equ. 178, 281-297 (2002)

15. Zhao, C, Zhou, S, Li, Y: Trajectory attractor and global attractor for a two-dimensional incompressible non-Newtonian fluid. J. Math. Anal. Appl. 325, 1350-1362 (2007)

16. Yuan, $\mathrm{H}, \mathrm{Xu}, \mathrm{X}$ : Existence and uniqueness of solutions for a class of non-Newtonian fluids with singularity and vacuum. J. Differ. Equ. 245, 2871-2916 (2008)

17. Málek, J, Nečas, J, Rokyta, M, Rúžička, M: Weak and Measure-Valued Solutions to Evolutionary PDEs. Chapman \& Hall, New York (1996)

18. Pokorný, M: Cauchy problem for the non-Newtonian viscous incompressible fluid. Appl. Math. 41, 169-201 (1996)

19. Shi, X: Some results of boundary problem of non-Newtonian fluids. Syst. Sci. Math. Sci. 9, 107-119 (1996)

20. Yin, $L, X u, X, Y$ uan, $H$ : Global existence and uniqueness of solution of the initial boundary value problem for a class of non-Newtonian fluids with vacuum. Z. Angew. Math. Phys. 59, 457-474 (2008)

21. Yuan, $\mathrm{H}, \mathrm{Li}, \mathrm{H}$ : Existence and uniqueness of solutions for a class of non-Newtonian fluids with vacuum and damping J. Math. Anal. Appl. 391, 223-239 (2012)

22. Rozanova, O: Nonexistence results for a compressible non-Newtonian fluid with magnetic effects in the whole space J. Math. Anal. Appl. 371, 190-194 (2010)

23. Qin, Y, Liu, X, Yang, X: Global existence and exponential stability of solutions to the one-dimensional full non-Newtonian fluids. Nonlinear Anal., Real World Appl. 13, 607-633 (2012)

24. Fang, D, Zi, R, Zhang, T: Global classical large solutions to a 1D fluid-particle interaction model: the bubbling regime. J. Math. Phys. 53, 033706 (2012)

25. Cho, Y, Choe, H, Kim, H: Unique solvability of the initial boundary value problems for compressible viscous fluids. J. Math. Pures Appl. 83, 243-275 (2004)

26. Cho, Y, Kim, H: Existence results for viscous polytropic fluids with vacuum. J. Differ. Equ. 228, 377-411 (2006) 